\title{
Retraction Note: Analysis of economic benefits of mineral resources development ecological environment based on ecological footprint
}

\author{
Rui Jia ${ }^{1} \cdot$ Liuyi Dai ${ }^{2,3}$
}

Published online: 13 December 2021

C) Saudi Society for Geosciences 2021

Retraction Note to: Arabian Journal of Geosciences (2021) 14: 1526 https://doi.org/10.1007/s12517-021-07711-1

The Editor-in-Chief and the Publisher have retracted this article because the content of this article is nonsensical. The peer review process was not carried out in accordance with the Publisher's peer review policy. Author Liuyi Dai disagrees with this retraction. Author Rui Jia has not responded to correspondence regarding this retraction.

The original article can be found online at https://doi.org/10.1007/ s12517-021-07711-1.

Liuyi Dai

liuyi_d@126.com

1 Department of Business Administration, Pusan National University, Busan 46241, Republic of Korea

2 Department of Economics, Pusan National University, Busan 46241, Republic of Korea

3 Baotou Teachers' College, Inner Mongolia University of Science and Technology, Baotou, Inner Mongolia 014030, People's Republic of China 